\title{
The C Terminus of Apocytochrome $b_{562}$ Undergoes Fast Motions and Slow Exchange among Ordered Conformations Resembling the Folded State ${ }^{\dagger}$
}

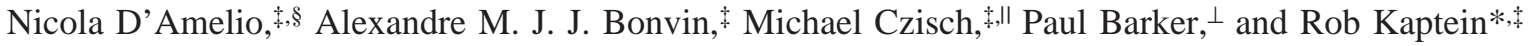 \\ NMR Department of the Bijvoet Center for Biomolecular Research, Utrecht University, Padualaan 8, \\ NL-3584 CH Utrecht, The Netherlands, and University Chemical Laboratory and Centre for Protein Engineering, \\ University of Cambridge, Lensfield Road, Cambridge CB2 1EW, U.K.
}

Received September 28, 2001; Revised Manuscript Received February 5, 2002

\begin{abstract}
The present work describes the dynamics of the apo form of cytochrome $b_{562}$, a small soluble protein consisting of 106 amino acid residues [Itagaki, E., and Hager, L. P. (1966) J. Biol. Chem. 241, 3687-3695]. The presence of exchange in the millisecond time scale is demonstrated for the last part of helix IV (residues 95-105 in the holo form). The chemical shift index analysis [Wishart, D. S., and Sykes, B. D. (1994) J. Biomol. NMR 4, 171-180] based on $\mathrm{H}_{\alpha}, \mathrm{C}_{\alpha}, \mathrm{C}_{\beta}$, and $\mathrm{C}^{\prime}$ chemical shifts suggests a larger helical content than shown in the NMR structure based on NOEs. These results indicate the presence of helical-like conformations participating in the exchange process. This hypothesis is consistent with amide deuterium exchange rates and the presence of some hydrogen bonds identified from amide chemical shift temperature coefficients [Baxter, N. J., and Williamson, M. P. (1997) J. Biomol. NMR 9, 359-369]. ${ }^{15} \mathrm{~N}$ relaxation indicates limited mobility for the amide protons of this part of the helix in the picosecond time scale. A 30 ns stochastic dynamics simulation shows small fluctuations around the helical conformation on this time scale. These fluctuations, however, do not result in a significant decrease of the calculated order parameters which are consistent with the experimental ${ }^{15} \mathrm{~N}$ relaxation data. These results resolve an apparent discrepancy in the NMR structures between the disorder observed in helix IV due to a lack of NOEs and the secondary structure predictions based on $\mathrm{H}_{\alpha}$ chemical shifts [Feng, Y., Wand, A. J., and Sligar, S. G. (1994) Struct. Biol. 1, 30-35].
\end{abstract}

Cytochrome $b_{562}$ is a small soluble protein consisting of 106 amino acid residues $(1,5)$ classified as an electrontransfer protein although its biological partner remains elusive (6). It is localized in the periplasm of Escherichia coli (7) and can be isolated from soluble extract of disrupted cells (1). The structure of ferricytochrome $b_{562}$ was determined by X-ray crystallography first at $2.5 \AA$ and later refined at $1.4 \AA$. The molecule is composed of four antiparallel helices (residues 3-19, 23-41, 56-80, and 85-105) with a short $3_{10}$ helix in the 15 -residue loop (residues 46-48) connecting the first and the second pairs of helices $(5,8,9)$. A $b$-type heme is ligated to the polypeptide chain through methionine 7 on the N-terminal helix and histidine 102 on the C-terminal helix (6). The solution structure of oxidized E. coli cytochrome $b_{562}$ does not show significant differences $(10,11)$, having a conformation intermediate between the two independent solid-state molecules.

\footnotetext{
This work was supported by the EU TMR Programme, Life Sciences Contract FMRX-CT98-0230, by the BBSRC (PDB) through an advanced fellowship, and by the Department of Chemistry, University of Perugia.

* To whom correspondence should be addressed. E-mail: kaptein@ nmr.chem.uu.nl.

Utrecht University.

$\S$ Present address: Department of Chemistry, University of Siena, via A. Moro, S. Miniato, 53100 Siena, Italy.

"Present address: Maz-Planck-Institut für Psychiatrie, Kraepelinstrasse 2, 80804 München, Germany.

${ }^{\perp}$ University of Cambridge.
}

The NMR structure of the apo form lacking the heme group reveals that the apoprotein has a topology similar to that of the holoprotein (4). The residues providing axial ligands to the heme iron in the holoprotein are, however, completely exposed to the solvent. In addition, the loop connecting helices II and III, the first three residues, and the last 12 residues are ill determined. Although considerable structural disorder is found in the fourth helix, the presence of some main-chain NOEs in residues 93-104, the deuterium exchange rates, and the small magnitude of the ${ }^{3} J_{\mathrm{HNH \alpha}}$ couplings support the helical character of this part of the structure (4). This is supported by molecular dynamics simulations (12) that suggest that all four helices present in the holo form are essentially intact and confirm that the difficulties in assigning helical NOEs in the C-terminal helix are not due to structural disorder. Deviations from random coil values of $\mathrm{H}_{\alpha}$ protons analyzed through the chemical shift index (2) support these results (4).

The stability of the apocytochrome has been probed through calorimetry, $\mathrm{GdmCl}$ denaturation, native state hydrogen exchange, and hydrogen exchange dependence on hydrostatic pressure $(13,14)$. Despite the variety of methods used in those studies, three main cooperative units were consistently found, the most stable one comprising helices II and III (residues 32-37, 70, 72, and 75-77), the other two units being formed by helix I (residues 13-19) and helix IV (residues 87-92) (14). These results suggest that the Nand C-terminal helices fold and unfold independently of the 
core of the molecule (13). The portion of helix IV identified as disordered in the NMR structure of the apo form shows, unlike the other more stable regions, a significant positive volume change at low pressure, suggesting the presence of a loosely packed open state at ambient pressure (14). 60\% and $80 \%$ helicity estimates were obtained from circular dichroism measurements for the apo and holo forms of cytochrome $b_{562}$, while NMR predicts an intermediate value of $71 \%$ for the apo form (15). Comparison of the free energies of stabilization and midpoints for the thermal transition of the apo- and holoproteins indicate that the protein is significantly stabilized upon binding heme (15). Interestingly, it has been shown that folding can be triggered by photoinduced electron transfer to unfolded Fe(III)cyt $b_{562}$ in 2-3 M guanidine hydrochloride solutions (16).

This study was undertaken to resolve the apparent discrepancy in the NMR structure between the disorder observed in helix IV due to a lack of NOEs and its predicted helical content based on $\mathrm{H}_{\alpha}$ chemical shifts (4). Our results demonstrate the presence of exchange in the millisecond time scale for the last part of helix IV (residues 95-106). Chemical shift index analysis (2) performed with four different nuclei $\left(\mathrm{H}_{\alpha}, \mathrm{C}_{\alpha}, \mathrm{C}_{\beta}, \mathrm{C}^{\prime}\right)$ suggests a larger helical content than shown in the NMR structure (4). This may indicate the presence of helical-like conformations participating in the exchange process. This hypothesis could explain both the measured amide deuterium exchange rates and the presence of some hydrogen bonds identified through amide chemical shift temperature coefficients (3). The ${ }^{15} \mathrm{~N}$ relaxation indicates restricted motions of the amide in the picosecond time scale in the C-terminal helix. Stochastic dynamics simulations (17) of the apo protein reveal for helix IV fluctuations around the helical conformation. These, however, do not result in a significant decrease of the calculated amide proton order parameters, which are in good agreement with the experimentally derived values.

\section{MATERIALS AND METHODS}

Sample Preparation. Apocytochrome $b_{562}$ was prepared as described for the holoprotein (10). E. coli strain BL21(DE3) mutant $\mathrm{C} 41$ (18) harboring plasmid pPB10 was grown in minimal media supplemented with $\left[{ }^{15} \mathrm{~N}\right] \mathrm{NH}_{4} \mathrm{Cl}$ and $\left[{ }^{13} \mathrm{C}\right]$ glucose $(4 \mathrm{~g} / \mathrm{L})$. Seventy percent of the expressed protein does not contain heme. The contents of the periplasm were extracted and combined with the culture medium, which had been previously concentrated by ultrafiltration using a 3000 MWCO Amicon membrane. This solution was exchanged into $20 \mathrm{mM}$ diethanolamine buffer, $\mathrm{pH} 8.5$, and sequentially purified by anion-exchange (Q-Sepharose), gel filtration (Superdex S-75), and anion-exchange (Mono-Q) chromatography, using $\mathrm{KCl}$ for gradients.

NMR Spectroscopy. NMR spectra were recorded on a Varian Inova 500 spectrometer equipped with a triple resonance probe with a shielded gradient coil. Higher sensitivity was achieved by the sensitivity enhancement scheme $(19,20)$ combined with gradient coherence selection $(20,21)$. Aliasing was used to increase the resolution in the nitrogen dimension. ${ }^{1} \mathrm{H}$ referencing was based on internal $\mathrm{TSP}^{1}$ with corrections for $\mathrm{pH}$ according to Cavanagh et al. (22). Indirect calibration was used for the other dimensions (22). Data were processed on Silicon Graphics O2 work- stations using NMRPipe (23), reduced, and analyzed with the in-house developed programs TRITON and REGINE, respectively. An apodization squared sine-bell function was applied, typically shifted by $\pi / 4$ in the ${ }^{1} \mathrm{H}$ dimension and $\pi / 2$ in the other dimensions.

Resonance Assignment and Secondary Structure Determination. Triple resonance experiments were performed on $1 \mathrm{mM}{ }^{13} \mathrm{C} /{ }^{15} \mathrm{~N}$-labeled protein in $90 \%{ }^{1} \mathrm{H}_{2} \mathrm{O} / 10 \% \mathrm{D}_{2} \mathrm{O}$ at 293 $\mathrm{K}$, containing $20 \mathrm{mM}$ phosphate at $\mathrm{pH}$ 5.1. A previous ${ }^{1} \mathrm{H}$ and ${ }^{15} \mathrm{~N}$ NMR assignment of apo $b_{562}$ was available at $\mathrm{pH}$ 5.1-5.2 and at $293 \mathrm{~K}(6) .{ }^{13} \mathrm{C}$ assignment of carbonyl CO, $\mathrm{C}_{\alpha}$, and $\mathrm{C}_{\beta}$ was achieved through $\mathrm{HNCO}(21,24,25,26)$, $\operatorname{HNCACB}(21,26,27)$, and $\mathrm{CBCA}(\mathrm{CO}) \mathrm{NH}(28,29)$. HBHA(CBCACO)NH allowed the determination of $\mathrm{H}_{\alpha}$ (in the case of adjacent residues missing amide protons ${ }^{1} \mathrm{H}$-TOCSY$\left[{ }^{1} \mathrm{H},{ }^{15} \mathrm{~N}\right]$-HSQC was used).

3D spectra were recorded typically using $1024 \times 100 \times$ 64 complex points in ${ }^{1} \mathrm{H},{ }^{13} \mathrm{C}$, and ${ }^{15} \mathrm{~N}$ dimensions, respectively, and processed with linear prediction and zero filling into spectra of $1024 \times 256 \times 128$ real data points. ${ }^{1} \mathrm{H}_{\alpha},{ }^{13} \mathrm{C}_{\alpha}$, ${ }^{13} \mathrm{C}_{\beta}$, and ${ }^{13} \mathrm{CO}$ chemical shifts were analyzed with the CSI program (PENCE/MCR Group Joint Software Centre) (2) to localize elements of secondary structure along the sequence.

Amide Deuterium Exchange and Temperature Dependence of ${ }^{1} \mathrm{H}$ Amide Chemical Shifts. ${ }^{15} \mathrm{~N}-\mathrm{HSQC}$ spectra (20) were recorded on a ${ }^{15} \mathrm{~N}$-labeled protein $1.89 \mathrm{mM}$ sample in $90 \%$ $\mathrm{H}_{2} \mathrm{O} / 10 \% \mathrm{D}_{2} \mathrm{O}$, containing $150 \mathrm{mM} \mathrm{KD} \mathrm{PO}_{4}$ at $\mathrm{pH} 5.10$. Water flip-back (30) was used for suppression of the solvent. The spectra were recorded at 278, 283, 288, 293, and $298 \mathrm{~K}$ using $2624 \times 200$ complex points in ${ }^{1} \mathrm{H}$ and ${ }^{15} \mathrm{~N}$ dimensions, respectively. Deuterium exchange spectra were recorded on the lyophilized sample dissolved in $\mathrm{D}_{2} \mathrm{O}$ at $\mathrm{pH} 5.3$ as measured after dissolution without taking into account the isotope effect (31). The first ${ }^{15} \mathrm{~N}-\mathrm{HSQC}$ spectrum was recorded $2 \mathrm{~min}$ and $40 \mathrm{~s}$ after dissolution. Five spectra were taken at 2 min intervals over the first 10 min after mixing (using only 1 scan and 40 complex points in the indirect dimension), then five more at 18 min intervals (4 scans and 100 complex points in the indirect dimension), followed by five more at $90 \mathrm{~min}$ intervals (20 scans and 100 complex points in the indirect dimension), and finally four spectra each taken at $7.5 \mathrm{~h}$ intervals (with 100 scans and 100 complex points in the indirect dimension). In all spectra 2624 complex points were used for the direct dimension. All spectra were processed with linear prediction in the indirect dimensions up to 256 points (150 for deuterium exchange experiments) and zero filled into spectra of $2048 \times 512$ real data points. ${ }^{1} \mathrm{H}$ amide shifts were plotted against temperature. The temperature coefficients were obtained from the slopes by linear fitting. Deuterium exchange data were plotted as peak heights (divided by the number of scans) against time and fitted utilizing the Levenberg-Marquardt algorithm for nonlinear least-squares minimization of the $\chi^{2}$ error function (32) using as a model function a three-parameter monoexponential decay. The resulting exponents were taken as a measure of the exchange rates. Plotting and fitting programs were provided by Dr. J. Craven, University of Sheffield.

\footnotetext{
${ }^{1}$ Abbreviations: CSI, chemical shift index; DEA, diethanolamine; $\mathrm{SD}$, stochastic dynamics; TSP, trimethylsilyl propionate.
} 
Relaxation Measurements. Relaxation experiments were performed on ${ }^{15} \mathrm{~N}$-labeled protein (see sample conditions above) according to $\mathrm{Li}$ and Montelione (33). Water presaturation was replaced by water flip-back (30), and the contribution from dipolar and CSA cross-correlation effects was suppressed by a series of $180^{\circ}$ pulses on hydrogen (34, $35) .{ }^{15} \mathrm{~N}$ decoupling during acquisition was performed using WALTZ16 $(36,37)$. The sequence used to determine $T_{2}$ is preceded by irradiation very far from the resonance $(100$ $\mathrm{kHz}$ ) to ensure that the same amount of rf power is dissipated into the sample for each relaxation delay. Relaxation delays used for determining longitudinal relaxation times were 32, $64,128,256,512,768,1024$, and 1536 ms. Relaxation delays used for determining transversal relaxation times were 8, 24, $32,40,48,64,96,144$, and $240 \mathrm{~ms}$. Peak volumes were used to determine intensities in each spectrum. Heteronuclear Overhauser enhancement factors $\eta=\left(I_{\mathrm{sat}}-I_{0}\right) / I_{0}$ were calculated from the normalized difference of peak volume in the presence $\left(I_{\mathrm{sat}}\right)$ and in the absence $\left(I_{0}\right)$ of ${ }^{1} \mathrm{H}$ saturation. In this experiment protons were saturated during $2.5 \mathrm{~s}$ prior to the pulse sequence in order to achieve steady-state intensities. The transmitter is arrayed alternatively in the middle of the amide proton region and off resonance in order to alternate FIDs with and without NOE effect. Relaxation times were obtained using an in-house written program which utilizes the Levenberg-Marquardt algorithm for nonlinear least-squares minimization of the $\chi^{2}$ error function (32) using a three-parameter monoexponential decay as a model function. The pulse sequence used to emphasize the exchange in the millisecond time scale only differs from the one used for determining ${ }^{15} \mathrm{~N} T_{2}$ by the frequency of repetition of $180^{\circ}$ pulses (and their number) (38). In the two experiments, the delays separating the 180 hard pulses were 4.8 and $1.6 \mathrm{~ms}$, and the global pulse train time was $9.6 \mathrm{~ms}$. All spectra were recorded using $1536 \times 160$ complex points in ${ }^{1} \mathrm{H}$ and ${ }^{15} \mathrm{~N}$ dimensions, respectively, and zero filled to $1024 \times 512$ real data points. The intensities were measured as peak heights.

Stochastic Dynamics Simulations. The protein was modeled according to the GROMOS96 model and force field 43B1 $(39,40)$. The starting structure was obtained from the crystallographic structure of the holoprotein (PDB entry 256B) (9) after removal of the heme group. The structure was first subjected to 250 steps of steepest descent energy minimization. The stochastic dynamics simulation was run for $30 \mathrm{~ns}$ using the GROMOS96 program $(39,41)$. The time step used in the leapfrog integration scheme was 0.002 ps. Covalent bond lengths were constrained with the procedure SHAKE (42) with a relative geometric tolerance of 0.0001 . The initial velocities were taken from a Maxwellian distribution at $300 \mathrm{~K}$. The reference temperature was set to $300 \mathrm{~K}$, but no coupling to a temperature bath was used since the temperature of the system is regulated by the stochastic forces. The nonbonded interaction pair list was updated every five steps with a cutoff of $0.8 \mathrm{~nm}$. The nonbonded interactions were calculated with group cutoffs using the twin-range method (43) with cutoffs of 0.8 and $1.4 \mathrm{~nm}$, respectively: all nonbonded interactions within $0.8 \mathrm{~nm}$ were calculated at every step while the long-range contribution, up to $1.4 \mathrm{~nm}$, was updated every five steps. Position restraints were applied on the solute heavy atoms during the first $100 \mathrm{ps}$ of the simulations with a constant of $500 \mathrm{~kJ} \mathrm{~mol}^{-1} \mathrm{~nm}^{-2}$. In stochastic dynamics simulations the interactions with the solvent are modeled implicitly by two additional forces: a frictional force fric proportional to the velocity and solventaccessible surface of the atoms to which it applies and a stochastic force $f^{\text {stoch }}$ following a Gaussian distribution and simulating the force the solute experiences due to collisions with solvent atoms (17). The atomic solvent-accessible surface areas were calculated as described in Yun-Yu et al. (44) and updated every 500 steps. Coordinates were saved every picosecond for analysis.

The secondary structure content of the protein was calculated using the SECSTR module of the program PROCHECK (45).

The order parameters $\left(S^{2}\right)$ of the $\mathrm{NH}$ vectors were calculated from the last $27.5 \mathrm{~ns}$ of the trajectory $(2.5-30.0$ ns) after least-squares fit of all $\mathrm{C}_{\alpha}$ atom positions on the initial structure as in Henry and Szabo (46)

$$
S^{2}=\frac{1}{2}\left(3 \sum_{\alpha=1}^{3} \sum_{\beta=1}^{3}\left\langle\mu_{\alpha} \mu_{\beta}\right\rangle^{2}-1\right)
$$

where $\mu_{\alpha}(\alpha=1,2,3)$ are the $x, y$, and $z$ components of the normalized interatomic vector in the molecular frame.

\section{RESULTS AND DISCUSSION}

${ }^{13} C_{\alpha},{ }^{13} C_{\beta}$, and ${ }^{13} \mathrm{CO}$ Resonance Assignment. Triple resonance experiments yielded the $\mathrm{C}_{\alpha}$ and $\mathrm{C}_{\beta}{ }^{13} \mathrm{C}$ shift resonances of all residues except for residues 45 and 105 . These data were used for the determination of secondary structure elements through the chemical shift index method. The complete set of resonance shifts for ${ }^{13} \mathrm{C}_{\alpha},{ }^{13} \mathrm{C}_{\beta}$, and ${ }^{13} \mathrm{CO}$ is deposited in the BMRB database (entry number 4767). The $\mathrm{C}_{\alpha}$ and $\mathrm{C}_{\beta}$ resonances of all prolines except residue 45 could be determined by connecting them to the following amide proton using a $3 \mathrm{D} \mathrm{CBCA}(\mathrm{CO}) \mathrm{NH}$ experiment. For proline 45 , which is followed by another proline, this was not possible. In the case of HNCO the carbonyl frequencies of residues preceding prolines $(44,45,52,55)$ and of the $C$ terminus (106) could not be determined. In addition, the $\mathrm{C}_{\alpha}$ resonance of residue 48 could not be determined due to the missing HN resonance of residue 49. Further, despite the larger dispersion offered by triple resonance experiments, the assignment of ${ }^{13} \mathrm{C}$ carbonyl frequencies of residues 94 and 96 remains unclear.

Secondary Structure Determination. Secondary structure elements such as $\alpha$-helices and $\beta$-sheets can be located through the analysis of ${ }^{1} \mathrm{H}$ and ${ }^{13} \mathrm{C}$ backbone chemical shifts (CSI index) (2). In the case of $\mathrm{H}_{\alpha}$, a negative deviation from random coil values indicates the presence of $\alpha$-helix structure while a positive deviation is typical for $\beta$-sheets. The contrary holds for $\mathrm{C}_{\alpha}$ and $\mathrm{CO}$ while positive deviations of $\mathrm{C}_{\beta}$ shifts indicate the presence of $\beta$-sheet structure. Results are shown in Figure 1. Although the shifts from random coil values appear to decrease in magnitude toward the $\mathrm{C}$ terminus, there is still evidence of $\alpha$-helical conformation. The CSI results (Figure 1e) for the apo form of cytochrome $b_{562}$ indicate the presence of helical conformation in positions 3-18, 23$41,57-80,85-95$, and $98-102$. These positions are quite similar to the helix position in the holoprotein (residues $3-19,23-40,56-80$, and 84-105) but disagree with the NMR structure of the apo form in which the last part of helix IV is missing. The fact that both $\mathrm{H}_{\alpha}$ and $\mathrm{C}_{\alpha}$ predictors 


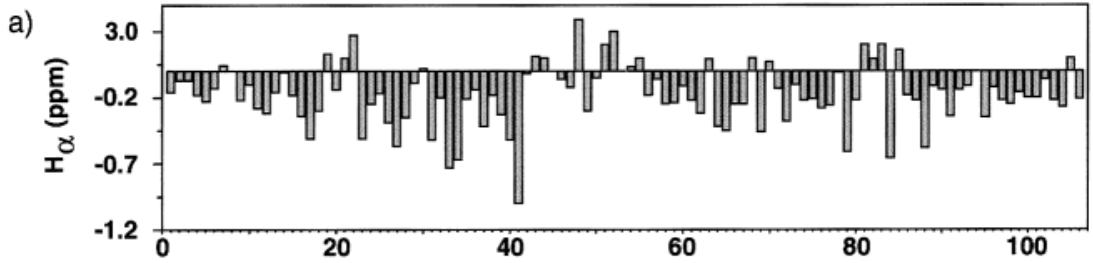

b)

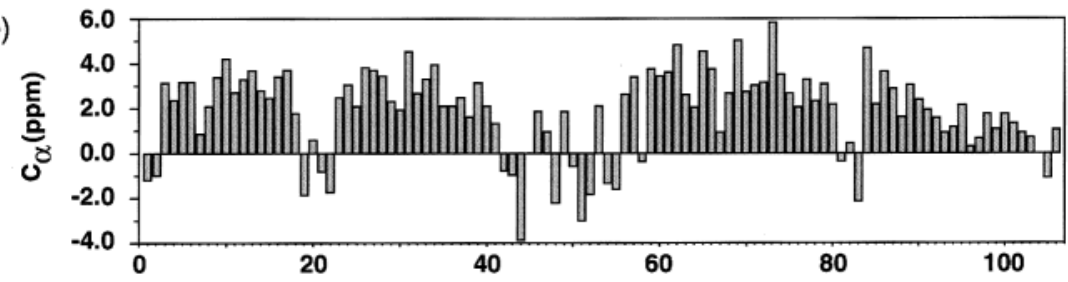

c)

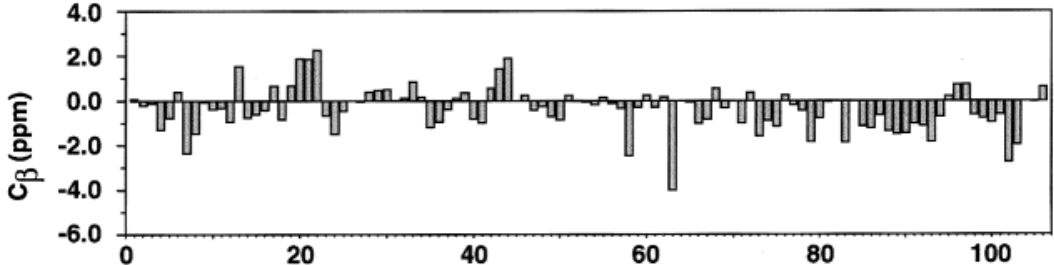

d)

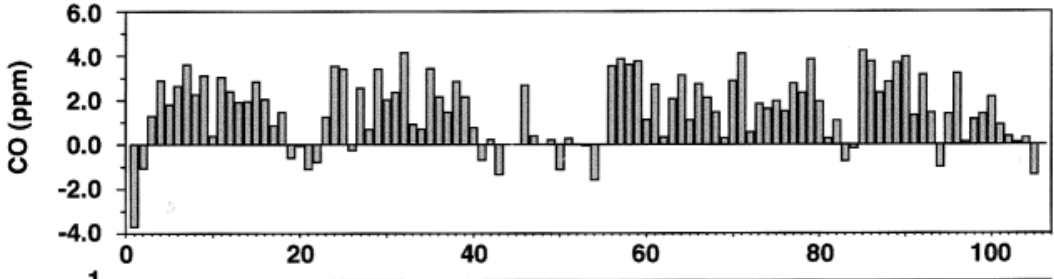

e)

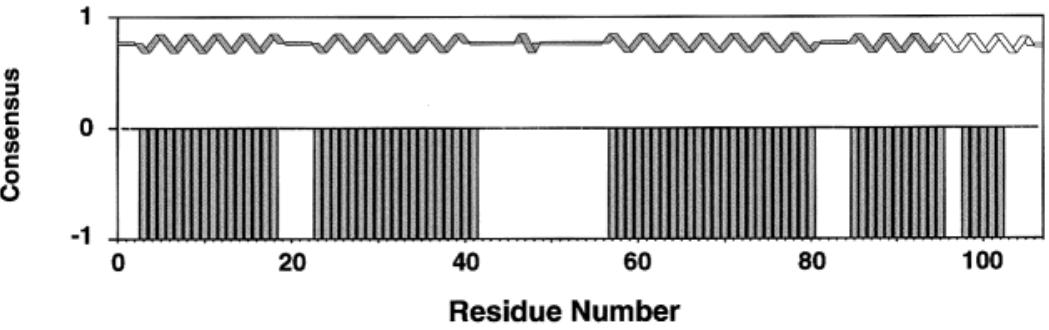

FIGURE 1: Chemical shift deviations from random coil values as a function of the residue number for (a) $\mathrm{H}_{\alpha}$, (b) $\mathrm{C}_{\alpha}$, (c) $\mathrm{C}_{\beta}$, (d) ${ }^{13} \mathrm{C}$ of carbonyl CO, and (e) corresponding secondary structure elements (consensus) as obtained from the CSI analysis. The consensus takes three possible values: -1 ( $\alpha$-helix), 0 (random coil or undetermined structure), and 1 ( $\beta$-sheet). The secondary structure elements of the holocytochrome $b_{562}$ are indicated as a cartoon in panel e with the part of the C-terminal helix lacking in the apo structure indicated with white instead of gray filling.

indicate helical content up to residue 102 is noteworthy, especially considering that they have the highest prediction accuracy (86\% and 84\%) (2). The presence of helical structure in the $\mathrm{C}$ terminus is further supported by the small magnitude of the ${ }^{3} J_{\mathrm{HN \alpha}}$ coupling constants (6). The presence of conformational exchange for this part of the protein could explain some of the discrepancies between the current results and the NMR structure of the apo form. A number of observations support this hypothesis:

(i) As compared to ordered helical structures in the protein, the segment from residue 95 onward shows a reduced peak intensity. This finding is not consistent with pure random coil mobility where stronger peaks would be expected.

(ii) Lowering the temperature results in a selective drop in peak heights from residue 95 to at least residue103, possibly consistent with a slowing of exchange processes to coalescence time scale at $500 \mathrm{MHz}$ (see Figure 4a).

(iii) Deviations of the chemical shifts from random coil values have borderline behavior which can originate from exchange among helical and nonhelical conformations. (iv) The lack of NOEs in 3D ${ }^{1} \mathrm{H}-{ }^{15} \mathrm{~N}$ NOESY-HSQC spectra can be explained by a lower sensitivity caused by exchange with nonhelical conformations.

Amide Deuterium Exchange and Temperature Dependence of ${ }^{1} H$ Amide Chemical Shifts. Deuterium exchange studies have already been performed (13); the experiments were, however, only initiated $20-25 \mathrm{~min}$ after addition of $\mathrm{D}_{2} \mathrm{O}$, and as a consequence, weak protection could not be revealed. The data reported here provide insight into exchange on shorter time scales since the first HSQC spectrum could be recorded after $2 \mathrm{~min}$ and $40 \mathrm{~s}$. Table 1 lists the amide proton exchange rates obtained as follows: peak heights were plotted against the delay time after dissolution in $\mathrm{D}_{2} \mathrm{O}$ buffer and fitted with monoexponential decays. Exchange rates were calculated as the exponents of the fitting curves.

The temperature dependence of the chemical shifts and intensities of backbone amide protons was analyzed in the range from 278 to $298 \mathrm{~K}$. Figure $2 \mathrm{~b}$ shows the amide chemical shift/temperature coefficient of all NHs that could be measured at least at two different temperatures. Temper- 


\begin{tabular}{|c|c|c|c|c|c|c|c|c|}
\hline $\begin{array}{c}\text { residue } \\
\text { no. }\end{array}$ & $\begin{array}{l}\text { ex- } \\
\text { change } \\
\text { rate }\end{array}$ & $\chi^{2}$ & $\begin{array}{c}\text { residue } \\
\text { no. }\end{array}$ & $\begin{array}{l}\text { ex- } \\
\text { change } \\
\text { rate }\end{array}$ & $\chi^{2}$ & $\begin{array}{c}\text { residue } \\
\text { no. }\end{array}$ & $\begin{array}{l}\text { ex- } \\
\text { change } \\
\text { rate }\end{array}$ & $\chi^{2}$ \\
\hline 2 & 0.387 & 0.101 & 36 & $<0.001$ & 0.070 & 74 & $e$ & \\
\hline 3 & 0.923 & 0.087 & 37 & $<0.001$ & 0.110 & 75 & $<0.001$ & 0.196 \\
\hline 4 & 1.489 & 0.737 & 38 & $<0.001$ & 0.220 & 76 & $<0.001$ & 0.277 \\
\hline 5 & 1.086 & 1.293 & 39 & 0.020 & 0.462 & 77 & $<0.001$ & 0.022 \\
\hline 6 & 0.557 & 0.048 & 40 & 0.023 & 0.705 & 78 & $<0.001$ & 0.067 \\
\hline 7 & $c$ & & 41 & 0.025 & 0.782 & 79 & $<0.001$ & 0.343 \\
\hline 8 & 1.137 & 0.533 & 42 & 0.113 & 0.480 & 80 & 0.030 & 0.514 \\
\hline 9 & $b$ & & 43 & 0.338 & 0.340 & 81 & 0.161 & 0.725 \\
\hline 10 & $c$ & & 44 & $b$ & & 82 & $b$ & \\
\hline 11 & 0.994 & 0.856 & 47 & $b$ & & 83 & $e$ & \\
\hline 12 & $b$ & & 48 & $d$ & & 84 & 0.748 & 0.611 \\
\hline 13 & 0.748 & 0.360 & 49 & & & 85 & 0.853 & 0.027 \\
\hline 14 & 0.183 & 1.551 & 50 & $b$ & & 86 & $c$ & \\
\hline 15 & 0.498 & 0.363 & 51 & $f$ & & 87 & 0.007 & 0.611 \\
\hline 16 & 0.116 & 0.875 & 52 & $b$ & & 88 & 0.006 & 0.141 \\
\hline 17 & 0.071 & 0.693 & 54 & $b$ & & 89 & $e$ & \\
\hline 18 & $c$ & & 55 & 0.684 & 2.000 & 90 & 0.004 & 0.193 \\
\hline 19 & 0.614 & 0.288 & 57 & $b$ & & 91 & 0.003 & 0.100 \\
\hline 20 & 0.684 & 1.380 & 58 & $d$ & & 92 & 0.103 & 0.885 \\
\hline 21 & $d$ & & 59 & $d$ & & 93 & 1.086 & 0.625 \\
\hline 22 & $b$ & & 60 & 0.045 & 0.006 & 94 & 0.071 & 0.667 \\
\hline 23 & $c$ & & 61 & 0.666 & 0.514 & 95 & 1.324 & 0.336 \\
\hline 24 & $e$ & & 62 & 0.798 & 0.480 & 96 & $d$ & \\
\hline 25 & 0.919 & 1.065 & 63 & $c$ & & 97 & 1.491 & 0.552 \\
\hline 26 & 0.045 & 0.447 & 64 & $b$ & & 98 & 0.082 & 0.360 \\
\hline 27 & $c$ & & 65 & $c$ & & 99 & 0.960 & 0.065 \\
\hline 28 & 0.124 & 0.511 & 66 & $f$ & & 100 & 0.084 & 0.142 \\
\hline 29 & 0.005 & 0.319 & 67 & 0.685 & 0.126 & 101 & $b$ & \\
\hline 30 & $<0.001$ & 0.020 & 68 & 0.021 & 0.269 & 102 & $d$ & \\
\hline 31 & $<0.001$ & 0.246 & 69 & $<0.001$ & 0.017 & 103 & 1.708 & 0.899 \\
\hline 32 & 0.027 & 0.317 & 70 & 0.021 & 0.313 & 104 & $d$ & \\
\hline 33 & $<0.001$ & 0.056 & 71 & 0.002 & 0.024 & 105 & $c$ & \\
\hline 34 & $<0.001$ & 0.033 & 72 & $<0.001$ & 0.029 & 106 & $b$ & \\
\hline 35 & 0.003 & 0.146 & 73 & $<0.001$ & 0.050 & & & \\
\hline
\end{tabular}

${ }^{a}$ The hydrogen exchange rates were measured by plotting peak heights $(I)$ against time $(t)$ and fitting the curve with a three-parameter monoexponential decay: $I=A+I_{0} \exp (-a t)$, where $I_{0}$ is the peak height immediately after dissolution in $\mathrm{D}_{2} \mathrm{O}, A$ corrects for baseline drifts, and $a$ is the exchange rate. ${ }^{b}$ Small: the signal was present in the first few spectra taken after dissolution, but poor signal to noise did not allow the measurement. ${ }^{c}$ Small: the signal was present in the first few spectra taken after dissolution, but severe overlap and poor resolution of spectra did not allow a good fit. ${ }^{d}$ The signal was not present in the first HSQC spectrum taken $2 \mathrm{~min}$ and $40 \mathrm{~s}$ after dissolution in $\mathrm{D}_{2} \mathrm{O} .{ }^{e}$ The signal was present in all spectra, but severe overlap or poor signal to noise did not allow the measurement. ${ }^{f}$ Severe overlap and poor resolution of spectra prevented the identification of the peak in all spectra.

ature dependence of the amide chemical shifts in conjunction with exchange data is a useful tool to localize hydrogenbonded amide protons along a sequence. In particular, an amide proton exchanging slowly and with a temperature coefficient more positive than $-4.5 \mathrm{ppb} / \mathrm{K}$ can be classified as hydrogen bonded (3). In addition, it has also been shown that a high negative value of temperature coefficient can originate from breaking of hydrogen bonds due to a loss of secondary structure on heating. The low negative value of some residues shown to exchange only relatively quickly with the solvent can be explained by population change upon temperature variation (47). This phenomenon can compete or add up to the general drift of chemical shifts toward low fields as the temperature increases, which has been explained by the loosening of hydrogen bonds (3). The latter case holds for residues presenting highly negative temperature dependence and measurable hydrogen exchange rate with the solvent. The phenomenon is more pronounced as the difference in chemical shifts between different conformations increases.

Focusing on helix IV and the $\mathrm{C}$ terminus, we observe that residues from position 87 to position 91 show temperature dependence consistent with exchange data only in the hypothesis of breaking of hydrogen bonds as the temperature increases. This effect is very pronounced for residue 87 , which resides at the beginning of the fourth helix. The structure seems to be quite ordered from position 92 to position 97 according to the temperature coefficients, but the exchange rates are quite large. This suggests that the chemical shift variation is no longer only depending on hydrogen bonding. From residue 98 up to residue 100 larger temperature dependences would suggest absence of hydrogen bonding. On the other hand, chemical exchange with $\mathrm{D}_{2} \mathrm{O}$ is not sufficiently fast to justify this hypothesis and matches with the previous data only in case of conformational exchange. Exchange-competent states due to helix end fraying have already been proposed for part of helix IV (residues 84-92) (13).

The temperature dependence of peak heights can also be indicative of conformational exchange. The relative peak height of each residue was monitored as the temperature was raised from 278 to $298 \mathrm{~K}$. Peak heights (each divided by the value of the first experiment) were plotted against temperature and fitted with a straight line. Temperature dependence was measured as the slope of each fitting curve. Although for some residues an exponential fitting would be more appropriate, linear fitting well expresses the behavior of most of the residues. In Figure 4a the dependence of peak height on temperature is expressed by the corresponding slopes as a function of the residue number. In the case of fast exchange, a drop in peak height is expected by lowering the temperature. This is clearly observed for the $\mathrm{C}$ terminus and, to a lesser extent, for residues 57 and 58. For very fast exchange, lowering the temperature by $10 \mathrm{~K}$ may be insufficient to reveal the exchange phenomena. The data show clear evidence of exchange near coalescence in the NMR time scale highly dependent on temperature. It is interesting to point out that only the amide protons of residues $2,4,12,21,25,44,47,52,57,59,63,85,87,100,101$, and 103 lie in the region between -6 to $-10 \mathrm{ppb} / \mathrm{K}$ (Figure $2 \mathrm{~b}$ ), where most of amide protons belonging to random coil conformations are found. If the possibility of breaking of hydrogen bonds with changing the temperature is taken into account (which can lead to highly negative temperature dependences even in the presence of hydrogen bonding), only amide protons of residues 12, 21, 44, 47, 52, 57, 59, and 101 (and probably 104) must be considered completely exposed, the others having measurable deuterium exchange rates. These residues reside in the middle part of helix I (12), in the first loop (21), in the big loop in positions flanking each one of the four prolines $(44,47,52,57)$, and in position 101 which is very near the end of helix IV according to the CSI method. Surprisingly, all residues at the $\mathrm{C}$ terminus except 96, 102, and 104 indicate some protection, showing the presence of some hydrogen bonding in the exchange equilibrium. The gap in the fourth helix at residues 96 and 97 in the chemical shift index is confirmed by deuterium exchange measurement that shows very fast exchange for residue 96. Residues 101 and 103 show a more 


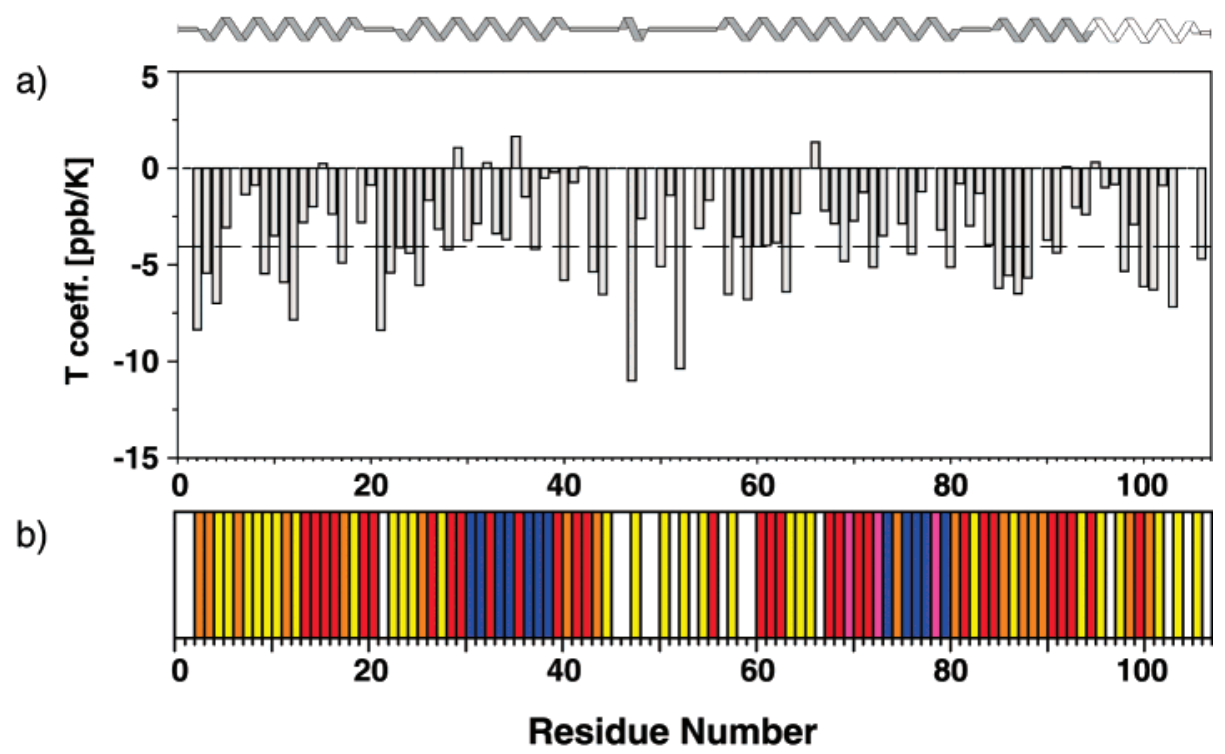

FIGURE 2: Amide ${ }^{1} \mathrm{H}$ chemical shift temperature coefficients (a) and qualitative hydrogen-bonding strength (b) versus residue number. The temperature coefficients were calculated as the slopes of a linear fitting of chemical shifts against temperature. Error bars were calculated through the error propagation formula, estimating a chemical shift uncertainty of $0.05 \mathrm{ppm}$. The critical value of $-4.5 \mathrm{ppb} / \mathrm{K}$ is shown by a dashed line. The color scheme for hydrogen-bonding strength is as follows: blue, very slow exchange and temperature coefficients larger than $-4.5 \mathrm{ppb} / \mathrm{K}$; magenta, very slow exchange and temperature coefficients smaller than $-4.5 \mathrm{ppb} / \mathrm{K}$; red, moderately fast exchange (rates between 0.001 and $1 \mathrm{~min}^{-1}$ ) and temperature coefficients larger than $-4.5 \mathrm{ppb} / \mathrm{K}$; orange, moderately fast exchange and temperature coefficients smaller than $-4.5 \mathrm{ppb} / \mathrm{K}$; yellow, all remaining residues present at least in the first HSQC spectrum. In cases when the temperature dependence data were absent, the classification was based on the deuterium exchange data alone, while in cases when the deuterium exchange values were absent, data were not classified. See caption of Figure 1 for an explanation of the secondary structure cartoon.

"random coil-like" behavior although their temperature coefficients lie in the very beginning of the random coil region, and deuterium exchange for residue 103 is only moderately fast. The presence of the last residue in the first HSQC spectrum of deuterium exchange data could be explained by an intraresidual hydrogen bonding with the terminal carboxyl group. A qualitative scheme showing the hydrogen bonds strengths is given in Figure 2c.

Relaxation Measurements. The ${ }^{15} \mathrm{~N}-{ }^{1} \mathrm{H}^{\mathrm{N}}$ heteronuclear NOE is a very sensitive probe for internal dynamics of the amide nitrogen and proton vectors (48) in the subnanosecond time scale. Figure 3a shows a ${ }^{15} \mathrm{~N}-{ }^{1} \mathrm{H}^{\mathrm{N}}$ heteronuclear NOE measured as a function of residue number. Empty slots in Figure 3 correspond to amide NH peaks whose integral could not be evaluated because of overlap (residues 6, 9, 10, 11, $13,18,24,27,29,34,39,48,51,58,59,62,63,65,66,74$, $78,79,89,94,95,97,99,104,105)$ or the absence of a HN amide peak $(1,45,46,49,53,56)$. With the exception of the last residue, apocytocrome $b_{562}$ does not show extensive subnanosecond mobility. Differences are, however, evident among different regions of the protein. Residues 2-5, 19$22,44-55$, and $81-83$, and the last part from at least residue 96 on, show enhanced mobility in the nano- to picosecond time scale. These regions clearly correspond to weak or loose parts of the protein as already identified in the previous measurements: the $\mathrm{N}$ terminus, the three loops, and the $\mathrm{C}$ terminus. The same information is evident in $T_{1}$ measurements (Figure 3b) which, although less sensitive, probe the spectral density also at the ${ }^{15} \mathrm{~N}$ frequency.

${ }^{15} \mathrm{~N} R_{2}$ relaxation rates (Figure $3 \mathrm{c}$ ) provide another probe for the dynamics of the protein backbone. It is sensitive to millisecond to microsecond processes. Conformational exchange, exchange with the solvent, or any time-dependent perturbation of the environment of a nucleus modulating its chemical shift causes magnetization to dephase on the transversal plane. Dephasing of magnetization leads to broadening or disappearance of the signal; since the acquisition time is in the order of hundreds of milliseconds, slow processes are able to efficiently dephase the transversal magnetization, resulting in faster apparent transverse relaxation rates (49). Although $R_{2}$ and $\eta$ depend on spectral densities at different frequencies, qualitatively, in the absence of slow perturbations, both quantities should be influenced only by motions in the nano- to picosecond time scale. In particular, for faster motions, low positive values of the transverse relaxation rate and high negative values of $\eta$ are expected. As a consequence, the values of $R_{2}$ and $\eta$ along the sequence should draw patterns of complementary shape. The deviation from this behavior may indicate the presence of exchange in the micro- to millisecond time scale. It is important to point out that the contribution of chemical exchange to relaxation is dependent on the rate of the exchange process, the chemical shift difference between participating sites, and the populations thereof (38). In particular, when the exchange rate $k_{\text {ex }}$ exceeds the difference in chemical shifts $\delta \omega$, a single resonance line is observed with an increased line width. As a consequence, some other processes can be active which are not evident in this comparison. For example, exchange between conformations exhibiting similar chemical shifts can be present but not visible in this analysis. Nevertheless, conformational exchange seems to be present for residues $2,3,4,17,19,21$, $22,26,44,47$, and 50 and for the $\mathrm{C}$ terminus, at least from residue 96 on. These results are confirmed by comparing the intensities of peaks in HSQC spectra implemented with CPMG-derived pulse trains with different repetition frequencies of the $180^{\circ}$ pulses (38) (see Materials and Methods). As shown in Figure 4b, exchange in the (sub)millisecond 


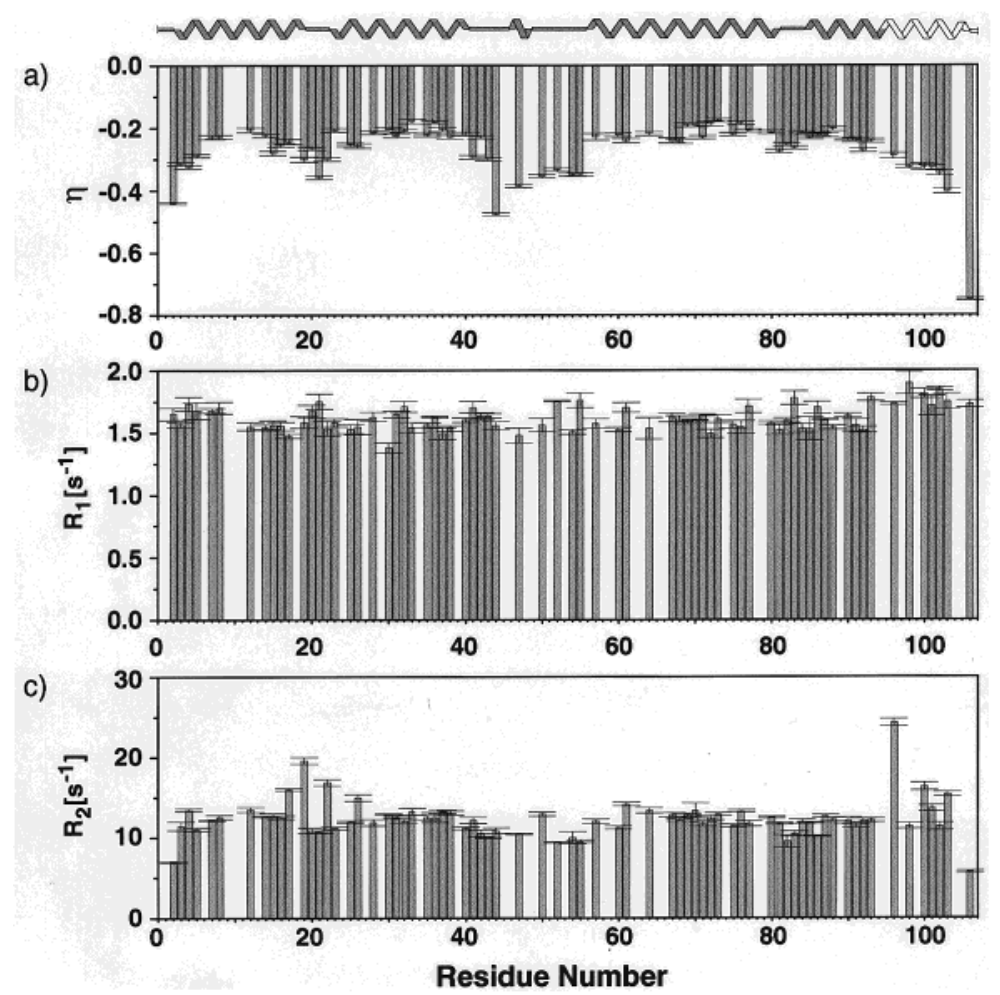

FIGURE 3: (a) Heteronuclear ${ }^{1} \mathrm{H}-{ }^{15} \mathrm{~N}$ NOEs measured as the fractional growth of peak intensity $\eta$ vs residue number. (b) Longitudinal relaxation rates $R_{1}$ vs residue number. (c) Transversal relaxation rates $R_{2}$ vs residue number. In all graphs empty slots correspond to residues for which the data could not be measured. Error bars were calculated using an estimate of the average noise. See caption of Figure 1 for an explanation of the secondary structure cartoon.

a)

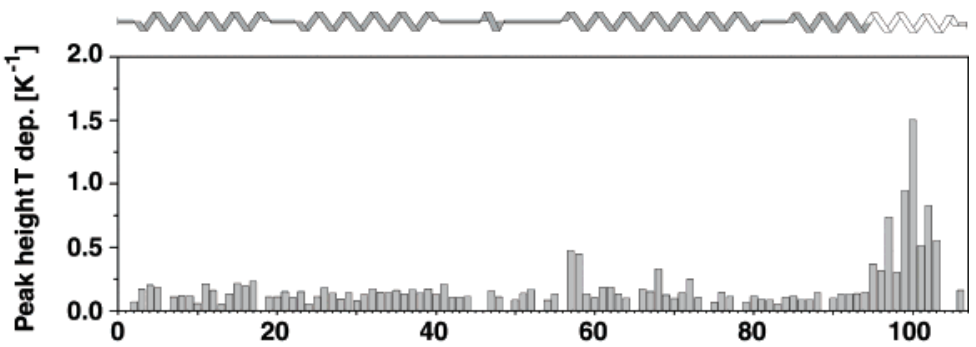

b)

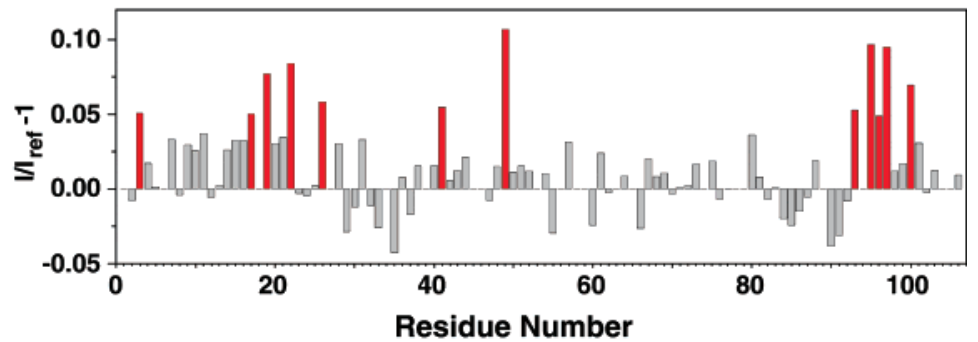

FIGURE 4: (a) Peak height temperature dependence vs residue number. (b) Peak height comparison between two HSQC spectra implemented with a CPMG sequence during indirect evolution. The two experiments differ in the pulse repetition delay of the CPMG sequence (4.8 and $1.6 \mathrm{~ms}$ ). Negative bars constitute a rough estimation of the error. Exchange contribution is evident for values larger than 0.04 (in red). See caption of Figure 1 for an explanation of the secondary structure cartoon.

time scale can be revealed by comparison of intensities in two spectra differing in the pulse repetition delay of the CPMG sequence (4.8 and $1.6 \mathrm{~ms}$ ). In the absence of exchange the ratio should be 1 ( 0 in the plot of Figure $4 \mathrm{~b}$ ). Greater values indicate regions of chemical exchange. Clearly, residues $17,19,22,26,41,49$, and from 93 to 100 present enhanced intensity (the assignment of residue 49 is only tenative). Again, the terminal part of helix IV shows exchange in the (sub)millisecond time scale although this is not evident for residues 98, 99, and from 101 on; these residues may exchange too fast with respect to the repetition rate of the refocusing pulses in the CPMG train. Actually, this experiment constitutes a sort of filter-selecting exchange in the order of the repetition rate. Since the exchange contribution to transversal relaxation time (which is inversely proportional to peak height) is dependent on pulse repetition rate $v_{\mathrm{CPMG}}$ only when $v_{\mathrm{CPMG}} \cong k_{\mathrm{ex}}$, we can estimate the lifetime of the conformations involved in the exchange process to be of the order of few milliseconds (repetition delays were 4.8 and $1.6 \mathrm{~ms}$ in the two experiments).

Stochastic Dynamics Simulation. To gain structural insight into the dynamic and hydrogen-bonding properties of apo- 


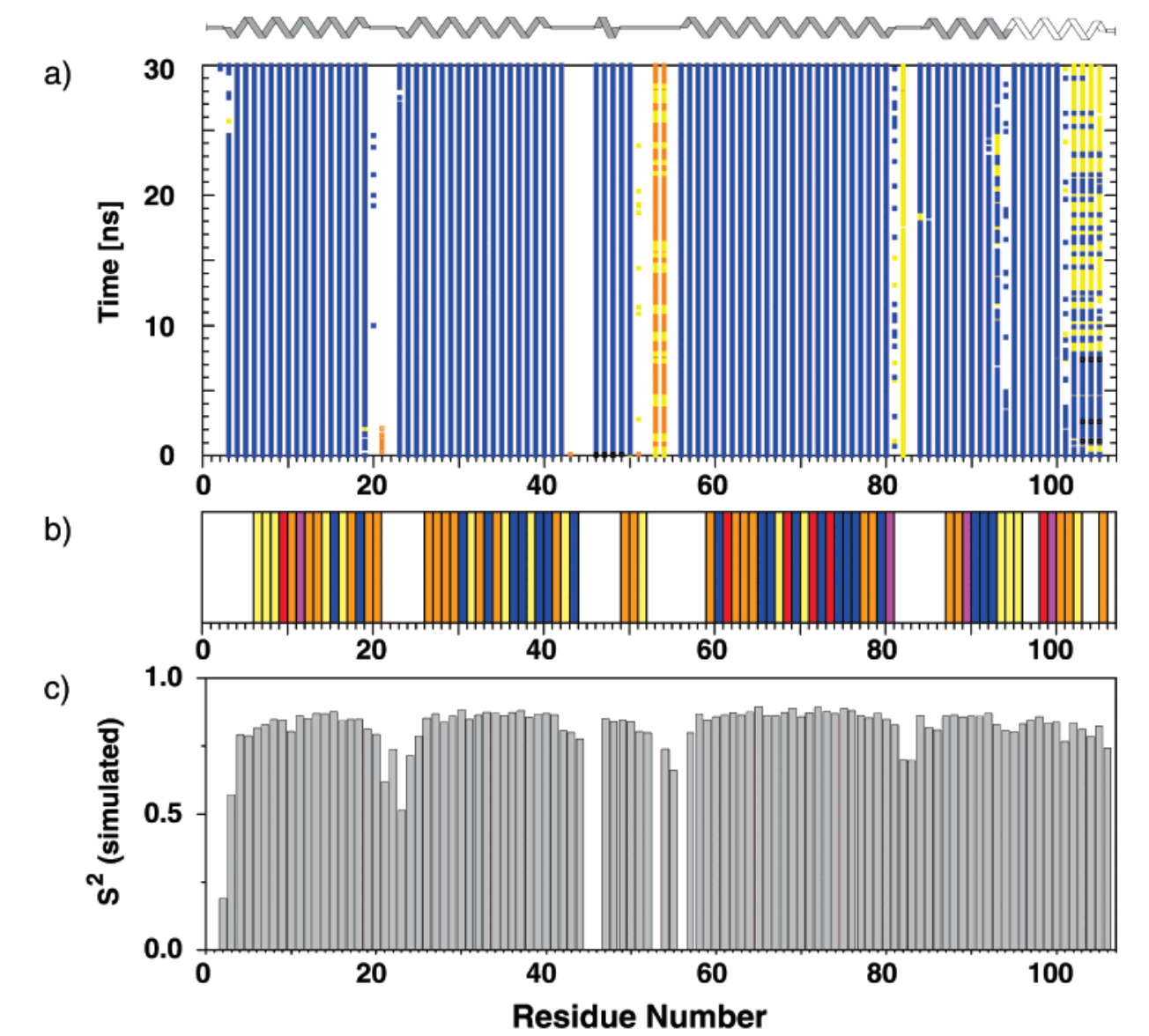

FIGURE 5: Secondary structure elements as a function of time (a), hydrogen bond lifetimes (b), and backbone amide order parameters (c) as calculated from the $30 \mathrm{~ns}$ stochastic dynamic simulation of apo $b_{562}$. The secondary structure elements are indicated as follows: blue, $\alpha$-helix; magenta, $3_{10}$ helix; yellow, hydrogen-bonded turn; orange, bend. The backbone $\mathrm{HN}_{i}-\mathrm{O}_{i-4}$ hydrogen bond lifetimes $\left(\tau_{\mathrm{HB}}\right)$ are colored as follows: blue, $\tau_{\mathrm{HB}}>15 \mathrm{~ns}$; magenta, $15 \mathrm{~ns}>\tau_{\mathrm{HB}}>10 \mathrm{~ns}$; red, $10 \mathrm{~ns}>\tau_{\mathrm{HB}}>6 \mathrm{~ns}$; orange, $6 \mathrm{~ns}>\tau_{\mathrm{HB}}>0.5 \mathrm{~ns}$; yellow, $\tau_{\mathrm{HB}}$ $<0.5 \mathrm{~ns}$. See caption of Figure 1 for an explanation of the secondary structure cartoon.

cytocrome $b_{562}$, a 30 ns stochastic dynamics simulation was performed starting from the X-ray structure of ferricytochrome $b_{562}$ after removal of the heme group. The choice of the holo structure as a starting point was motivated by the experimental evidence for the presence of the last helix in solution. A previous MD study by Laidig and Dagget (16) compared the holo- and apocytocrome structures. Those simulations (both starting from the holo structures) were run in explicit water for up to $1.8 \mathrm{~ns}$. The apocytocrome structure was described as a molten globule with increased side-chain mobility. This work already pointed toward the presence of the last helix. Here we chose to treat the solvent implicitly using stochastic dynamics in order to reach longer time scales for comparison with the NMR relaxation and hydrogen exchange data. Our simulation deviates from the starting structure (the holoprotein from which the heme has been removed) to reach a plateau around $0.3 \mathrm{~nm}$ after approximately $2 \mathrm{~ns}$. This is slightly larger than the $0.25 \mathrm{~nm}$ rmsd value reported by Laidig and Dagget (16). The system does, however, not move closer to the apocytocrome structure (PDB entry 1APC) and fluctuates at about $0.4 \mathrm{~nm}$ backbone rmsd from it. The C-terminal helix in the NMR solution structure was poorly defined due to overlap and motional averaging (4). As was the case in the previous MD work, the last helix is in major part maintained throughout the 30 ns simulation with some breaks around residue 94. The evolution of the secondary structure elements as a function of the simulation time is reported in Figure 5a. The largest variations are localized in the $\mathrm{C}$ terminus. Starting from the structure of the holo form where the fourth helix reaches residue 105, we observe a fluctuation around a helical conformation starting from residue 101 onward, a value in good agreement with the result of the chemical shift index (in which the last helix ends with residue 102) especially considering the difficulty in defining the exact position of both ends of an helix. To obtain some more insight in the weakest part of the structure, at least from a kinetic point of view, the average lifetimes of $\mathrm{N}_{i}-\mathrm{H}-\mathrm{O}_{i-4}$ hydrogen bonds were analyzed (Figure $5 \mathrm{~b}$ ). The resulting picture resembles the qualitative scheme presented in Figure $2 \mathrm{c}$ based on exchange data and amide shift temperature coefficients. Focusing on the $\mathrm{C}$ terminus, we can see that the simulation describes the experimental data very well. We observe in fact a gap in the fourth helix around the positions that were shown to exchange very fast in the deuterium exchange experiments (residues 95 and 96). Furthermore, a helical conformation continues up to the end, but as suggested by the CSI method, its persistence decreases around residue 102. The amide vector internal correlation functions and the corresponding order parameters were calculated from the last $27.5 \mathrm{~ns}$ of the simulation (Figure 5c). Note that this order parameter does not reflect bond fluctuations since it was calculated from normalized internuclear vectors. The calculated order parameters indicate only limited subnanosecond motion in the terminal helix, which is consistent with experimental relaxation data $\left(R_{1}\right.$ and $\left.\mathrm{NOE}\right)$. 


\section{CONCLUSIONS}

The removal of the heme destabilizes apocytochrome $b_{562}$ significantly at $\mathrm{pH} 5$ and room temperature. In the picosecond time scale, enhanced mobility is present in the loop connecting helices II and III and in the terminus of helix IV. For the latter, extensive motions are associated with low values of the order parameters, indicating a very disordered region in agreement with the poor definition in the NMR structure. The picture coming out from the analysis of amide deuterium exchange data and temperature dependence of chemical shifts shows a molecule with a wide variation in the strength of the hydrogen bond interactions stabilizing the structure. Data are consistent with the presence of both very strong and very weak hydrogen bonds and with conformational exchange mostly localized in the first and the fourth helix. Millisecond time scale conformational exchange (with coalescence near $283 \mathrm{~K}$ ) was demonstrated mostly located at the ends of structured parts but especially around the first loop and at the $\mathrm{C}$ terminus with strong suggestions of the presence of helical-like conformations participating in the exchange process. Our results confirm the previous NMR observations indicating the presence of helix conformations in the C-terminal part of apocytocrome based on the $\mathrm{H}_{\alpha}$ chemical shifts and the small magnitude of the $\mathrm{H}_{\alpha}-\mathrm{H}_{\mathrm{N}} J$-couplings (4). The emerging model is one in which the last segment of helix IV undergoes conformational exchange on an intermediate time scale, resulting in a decrease of the peak intensities. This explains the lack of NOEs leading to the structural disorder of helix IV in the previous structural study of apocytochrome $b_{562}$ (4). Significant helical population is, however, maintained as indicated by the chemical shift index, amide exchange data, and temperature coefficients.

\section{ACKNOWLEDGMENT}

We thank Dr. Jeremy Craven for helpful discussion and assistance in deuterium exchange experiments.

\section{SUPPORTING INFORMATION AVAILABLE}

One table listing ${ }^{1} \mathrm{H}_{\alpha}$ and ${ }^{13} \mathrm{C}_{\alpha},{ }^{13} \mathrm{C}_{\beta}$ chemical shifts of apocytochrome $b_{562}$ at $\mathrm{pH} 5.1$ and temperature $293 \mathrm{~K}$. This material is available free of charge via the Internet at http:// pubs.acs.org.

\section{REFERENCES}

1. Itagaki, E., and Hager, L. P. (1966) J. Biol. Chem. 241, 36873695.

2. Wishart, D. S., and Sykes, B. D. (1994) J. Biomol. NMR 4, $171-180$.

3. Baxter, N. J., and Williamson, M. P. (1997) J. Biomol. NMR 9, 359-369.

4. Feng, Y., Wand, A. J., and Sligar, S. G. (1994) Struct. Biol. $1,30-35$.

5. Lederer, F., Glatigny, A., Bethge, P. H., Bellamy, H. D., and Mathews, F. S. (1981) J. Mol. Biol. 148, 427-448.

6. Feng, Y., Wand, A. J., and Sligar, S. G. (1991) Biochemistry 30, 7711-7717.

7. Nikkila, H., Gennis, R. B., and Sligar, S. G. (1991) Eur. J. Biochem. 202, 309-313.

8. Mathews, F. S., Bethge, P. H., and Czerwinski, E. W. (1979) J. Biol. Chem. 254, 1699-1760.
9. Hamada, K., Bethge, P. H., and Mathews, F. S. (1995) J. Mol. Biol. 247, 947-962.

10. Arnesano, F., Banci, L., Bertini, I., Faraone-Mennella, J., Rosato, A., Barker, P. D., and Fersht, A. R. (1999) Biochemistry 38, 8657-8670.

11. Arnesano, F., Banci, L., Bertini, I., Van Der Wetering, K., Czisch, M., and Kaptein, R. (2000) J. Biomol. NMR 17, 295304.

12. Laidig, K. E., and Dagget, V. (1996) Folding Des. 1, 335346.

13. Fuentes, E. J., and Wand, A. J. (1998) Biochemistry 37, 36873698.

14. Fuentes, E. J., and Wand, A. J. (1998) Biochemistry 37, 98779883.

15. Feng, Y., and Sligar, G. (1991) Biochemistry 30, 1015010155.

16. Wittung-Stafshede, P., Lee, J. C., Winkler, J. R., and Gray, H. B. (1999) Proc. Natl. Acad. Sci. U.S.A. 96, 6587-6590.

17. van Gunsteren, W. F., Berendsen, H. J. C., and Rullmann, J. A. C. (1981) Mol. Phys. 44, 69-95.

18. Miroux, B., and Walker, J. E. (1996) J. Mol. Biol. 260, 289298.

19. Cavanagh, J., Palmer, A. G., III, Wright, P. E., and Rance, M. (1991) J. Magn. Reson. 91, 429-436.

20. Kay, L. E., Keifer, P., and Saarinen, T. (1992) J. Am. Chem. Soc. 114, 10663-10665.

21. Muhandiram, D. R., and Kay, L. E. (1994) J. Magn. Reson. B103, 203-216.

22. Cavanagh, J., Fairbrother, W. J., Palmer, A. G., III, and Skelton, N. J. (1996) Protein NMR Spectroscopy: Principles and practice, Academic Press, New York.

23. Delaglio, F., Grzesiek, S., Vuister, G. W., Zhu, G., Pfeifer, J., and Bax, A. (1995) J. Biomol. NMR 6, 277-293.

24. Ikura, M., Kay, L. E., and Bax, A. (1990) Biochemistry 29, 4659-4667.

25. Grzesiek, S., and Bax, A. (1992) J. Magn. Reson. 96, 432440.

26. Kay, L. E., Xu, G. Y., and Yamazaki, T. (1994) J. Magn. Reson. A109, 129-133.

27. Wittekind, M., and Mueller, L. (1993) J. Magn. Reson. B101, 201-205.

28. Grzesiek, S., and Bax, A. (1992) J. Magn. Reson. 114, 62916293.

29. Grzesiek, S., and Bax, A. (1993) J. Biomol. NMR 3, 185204.

30. Grzesiek, S., and Bax, A. (1993) J. Am. Chem. Soc. 115, $12593-12594$.

31. Glasoe, K., and Long, F. A. (1960) J. Phys. Chem. 64, 188190.

32. Press, W. H., Teukolsky, S. A., Vettering, W. T., and Flannery B. P. (1992) Numerical Recipes, Cambridge University Press, Cambridge, U.K.

33. Li, Y., and Montelione, G. (1995) Biochemistry 34, $2048-$ 2423.

34. Palmer, A. G., III, Skelton, N. J., Chazin, W. J., Wright, P. E., and Rance, M. (1992) Mol. Phys. 75, 699-711.

35. Kay, L. E., Nicholson, L. K., Delaglio, F., Bax, A., and Torchia, D. A. (1992) J. Magn. Reson. 97, 359-375.

36. Shaka, A. J., Keeler, J., Frenkiel, T., and Freeman, R. (1983) J. Magn. Reson. 52, 335-338.

37. Shaka, A. J., Keeler, J., and Freeman, R. (1983) J. Magn. Reson. 53, 274-293.

38. Mulder, F. A., van Tilborg, P. J., Kaptein, R., and Boelens, R. (1999) J. Biomol. NMR 13, 275-288.

39. van Gunsteren, W. F., Billeter, S. R., Eising, A. A., Hünenberger, P. H., Krüger, P., Mark, A. E., Scott, W. R. P., and Tironi, I. G. (1996) Biomolecular Simulation: The GROMOS96 Manual and User Guide, pp 1-1042, Vdf Hochschulverlag AG an der ETH Zürich, Zürich, Switzerland.

40. Daura, X., Mark, A. E., and van Gunsteren, W. F. (1998) J. Comput. Chem. 19, 535-547.

41. Scott, W. R. P., Hünenberger, P. H., Tironi, I. G., Mark, A. E., Billeter, S. R., Fennen, J., Torda, A. E., Huber, T., Krüger, 
P., and van Gunsteren, W. F. (1999) J. Phys. Chem. A 103, 3596-3607.

42. Ryckaert, J.-P., Ciccotti, G., and Berendsen, H. J. C. (1977) J. Comput. Phys. 23, 327-341.

43. van Gunsteren, W. F., and Berendsen, H. J. C. (1990) Angew. Chem., Int. Ed. Engl. 29, 992-1023.

44. Yun-Yu, S., Lu, W., and van Gunsteren, W. F. (1988) Mol. Simul. 1, 369-383.

45. Laskowski, R. A., MacArthur, M. W., and Thornton, J. M. (1993) J. Appl. Crystallogr. 26, 283-291.
46. Henry, E. R., and Szabo, A. (1985) J. Chem. Phys. 82, 375390.

47. Andersen, N. H., Chen, C., Marschner, T. M., Krystek, S. R., Jr., and Bassolino, D. A. (1992) Biochemistry 31, 1280-1295.

48. Kay, L. E., Torchia, D. A., and Bax, A. (1989) Biochemistry 28, 8972-8979.

49. Palmer, A. G., III, Williams, J., and McDermott, A. (1996) J. Phys. Chem. 100, 13293-13310.

BI011863N 